www.nature.com/jhg

\title{
Deletion at chromosome 10p11.23-p12.1 defines characteristic phenotypes with marked midface retrusion
}

\author{
Nana Okamoto ${ }^{1,2}$, Shin Hayashi ${ }^{1,3}$, Ayako Masui ${ }^{4}$, Rika Kosaki ${ }^{5}$, Izumi Oguri ${ }^{6}$, Tomoko Hasegawa ${ }^{7}$, \\ Issei Imoto $^{8}$, Yoshio Makita ${ }^{9}$, Akira Hata ${ }^{10}$, Keiji Moriyama ${ }^{2,3}$ and Johji Inazawa ${ }^{1,3,11}$
}

Approximately $3 \%$ of the live-born infants have major dysmorphic features, and about two-thirds of which are observed in the maxillofacial region; however, in many cases, the etiology of the dysmorphic features remains uncertain. Recently, the genome-wide screening of large patient cohorts with congenital disorders has made it possible to discover genomic aberrations corresponding to the pathogenesis. In our analyses of more than 536 cases of clinically undiagnosed multiple congenital anomalies and mental retardation (MR) by microarray-based comparative genomic hybridization, we detected two non-consanguineous unrelated patients with microdeletions at 10p11.23-p12.1, which overlapped for $957 \mathrm{~kb}$, including four protein-coding genes: $A R M C 4, M P P 7, W A C$ and $B A M B I$. As the two patients had similar phenotypes; for example, MR and multiple maxillofacial abnormalities including midface retrusion, wide mouth and large tongue, we assessed the phenotypes in detail to define the common features, using quantitative evaluations of the maxillofacial dysmorphism. The concordance of the genetic and phenotypic alterations is a good evidence of a new syndrome. Although an interstitial deletion of 10p is rare, the current study is the first trial to examine precisely the craniofacial characteristics of patients with a heterozygous deletion at 10p11.23-p12.1, and presents good evidence to diagnose potential patients with the same genetic cause.

Journal of Human Genetics (2012) 57, 191-196; doi:10.1038/jhg.2011.154; published online 19 January 2012

Keywords: array CGH; developmental retardation; large tongue; midface retrusion; wide mouth; 10p interstitial deletion

\section{INTRODUCTION}

Approximately $3 \%$ of the live-born infants have major dysmorphic features, about two-thirds of which are observed in the maxillofacial region, which potentially cause medical, social, cosmetic and psychological problems, although the etiology of a significant proportion of such cases remains unclear. ${ }^{1-4}$ Recently, array-based comparative genome hybridization $(\mathrm{aCGH})$ has been employed for the diagnosis of patients with congenital disorders; for example, developmental delay/mental retardation (MR) and/or multiple congenital anomalies (MCA) in place of conventional karyotyping. ${ }^{5}$ We have analyzed more than 500 patients with MCA and MR of unknown etiology using in-house bacterial artificial chromosome (BAC) arrays, including dysmorphic features in maxillofacial region, and assessed the pathogenicity of copy-number variants $(\mathrm{CNVs}) .{ }^{6}$ Through the screening, we detected several cases with overlapping CNVs and similar phenotypes, which may potentially define a new syndrome. Among such cases, we detected two patients with common phenotypes including characteristic maxillofacial dysmorphisms with overlapping deletions at 10p11.23-p12.1. The concordance of phenotypic and genetic alterations in these two cases seems to provide evidence for establishing a new syndrome. Patients with an interstitial deletion at around 10p11.23-p12.1 have been rarely reported, although the deletion and severity of symptoms are variable., ${ }^{7,8}$ Thus, we evaluated in detail both the genomic aberrations and the clinical features including skeletal characteristics of the patients, and manifested a value of characteristic maxillofacial features defining a new syndrome corresponding with a deletion at 10p11.23-p12.1.

\section{MATERIALS AND METHODS}

We constructed a consortium with 23 medical institutes and hospitals in Japan, and had recruited more than 536 patients with MCA/MR clinically

\footnotetext{
${ }^{1}$ Department of Molecular Cytogenetics, Medical Research Institute and School of Biomedical Science, Tokyo Medical and Dental University, Tokyo, Japan; ${ }^{2}$ Department of Maxillofacial Orthognathics, Tokyo Medical and Dental University, Tokyo, Japan; ${ }^{3}$ Hard Tissue Genome Research Center, Tokyo Medical and Dental University, Tokyo, Japan; ${ }^{4}$ Department of Pediatrics, Yaizu City Hospital, Yaizu, Japan; ${ }^{5}$ Division of Medical Genetics, National Center for Child Health and Development, Tokyo, Japan; ${ }^{6}$ Department of Pediatrics, Shizuoka Saiseikai General Hospital, Shizuoka, Japan; ${ }^{7}$ Genetic Support and Consultation Office, Tokyo, Japan; ${ }^{8}$ Department of Human Genetics and Public Health, Institute of Health Biosciences, The University of Tokushima Graduate School, Tokushima, Japan; ${ }^{9}$ Department of Pediatrics, Asahikawa Medical College, Hokkaido, Japan; ${ }^{10}$ Department of Public Health, Chiba University Graduate School of Medicine, Chiba, Japan and ${ }^{11}$ Global Center of Excellence (GCOE) Program for 'International Research Center for Molecular Science in Tooth and Bone Diseases', Tokyo Medical and Dental University, Tokyo, Japan

Correspondence: Professor J Inazawa, Department of Molecular Cytogenetics, Medical Research Institute and School of Biomedical Science, Tokyo Medical and Dental University, 1-5-45 Yushima, Bunkyo-ku, Tokyo 113-8510, Japan.

E-mail: johinaz.cgen@mri.tmd.ac.jp
}

Received 15 November 2011; revised 14 December 2011; accepted 26 December 2011; published online 19 January 2012 
undiagnosed by an expert in medical genetics or a dysmorphologist. ${ }^{6}$ All showed a normal karyotype with approximately $400-550$ bands on G-banding karyotyping. Among the analyses, we detected the following two patients.

This study was approved by the local ethics committee and all the institutions involved in this project. All subjects provided written informed consent for the use of their phenotypic and genetic data.

\section{Clinical report}

Patient 1 was a 7-year, 10-month-old Japanese girl, born to healthy parents by normal vaginal delivery (Figure 1a, Table 1). Her younger brother was healthy. Her psychomotor development was retarded; she first raised her neck at 8 months, rolled over at 21 months, sat unaided at 24 months and stood with support at 26 months. Her developmental quotient was 36 at the age of 2 years. She showed several characteristic dysmorphic features, especially in the maxillofacial area; flat occiput, frontal bossing, midface retrusion, coarse face, downslanted palpebral fissures, synophrys (apparent in the lateral half), deep set eyes, epicanthus, anteverted nares, wide nasal bridge, malformed auricles, wide mouth, absent Cupid's bow, everted vermilion of the upper lip, downturned corners of the mouth and large tongue (Figure 1a). She also had several cardiac abnormalities (Table 1). Brain computed tomography showed no abnormality. Conventional karyotyping of her peripheral blood lymphocytes with approximately 400-550 bands revealed a normal female karyotype, 46,XX.

Patient 2 was a 6-year, 8-month-old Japanese boy, born to healthy parents by normal vaginal delivery (Figure 1b, Table 1). His elder brother was healthy. His psychomotor development was moderately retarded; he raised his neck at 10 months, and at present, can speak several words. He also showed several dysmorphic features in the maxillofacial area; frontal bossing, midface retrusion, coarse face, downslanted palpebral fissures, synophrys (especially in the lateral half), deep set eyes, epicanthus, wide nasal bridge, bulbous nose, wide mouth, absent Cupid's bow, everted vermilion of the upper lip, downturned corners of the mouth and large tongue (Figure 1b). He was diagnosed with a short stature and growth hormone deficiency. Brain magnetic resonance imaging showed no abnormality at that time. Conventional karyotyping of his peripheral blood lymphocytes with approximately $400-550$ bands revealed a normal male karyotype, 46,XY.

\section{DNA extraction}

Genomic DNA and metaphase chromosomes were prepared from peripheral blood obtained with prior written informed consent from the parents. Genomic DNA as a normal control was obtained from a lymphoblastoid cell line of one healthy male and one healthy female as used in our previous study. ${ }^{6}$

\section{aCGH analysis}

We applied an in-house BAC-based array, the 'MCG Whole Genome Array4500', which covers all 24 human chromosomes with 4523 BACs at intervals of approximately $0.7 \mathrm{Mb},{ }^{9}$ and also an oligonucleotide array (Agilent Human Genome CGH Microarray 244K; Agilent Technologies, Santa Clara, CA, USA). Hybridization and analysis were performed as described elsewhere. ${ }^{6}$
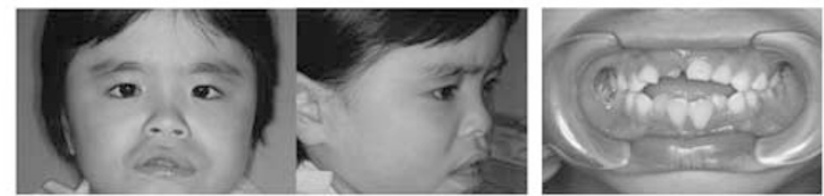
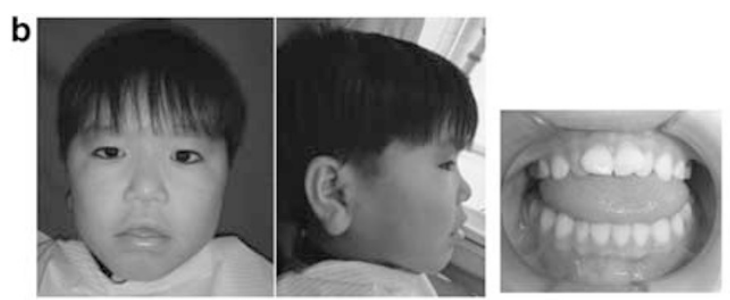

C
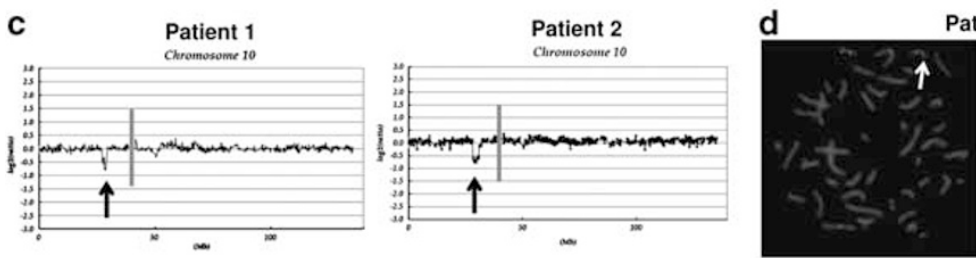

Patient 1
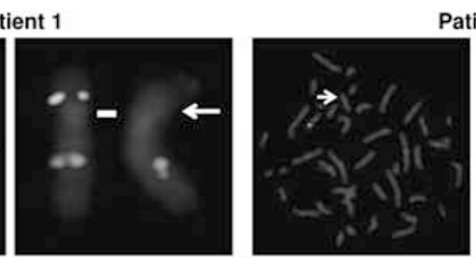

Patient 2

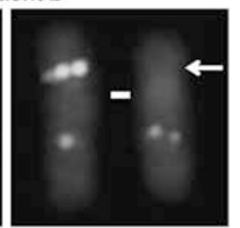

e

$\mathbf{f}_{\text {tel }} \frac{25}{+}$

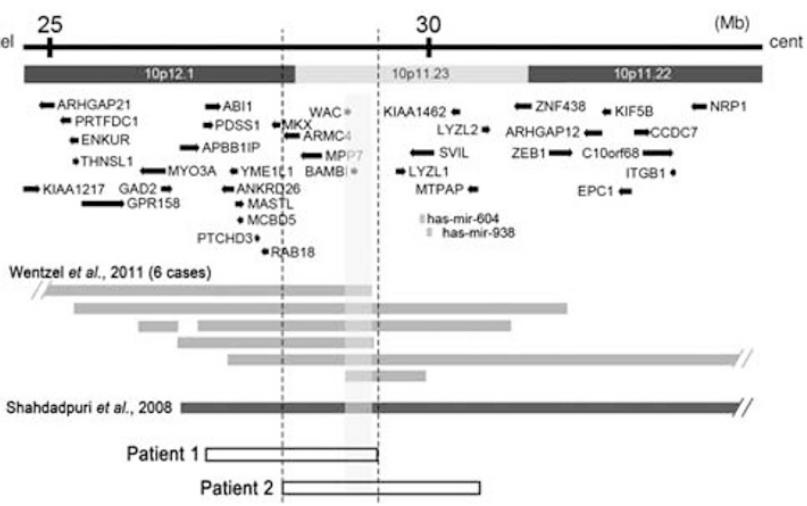

Figure 1 Clinical manifestations and cytogenetic analyses of the patients. (a) Facial and oral features of patient 1. (b) Facial and oral features of patient 2. (c) Results of the Whole Genome Array-4500 analyses in patient 1 and patient 2. Black arrows indicate each CNV and the grey vertical bars indicate the position of a centromere. (d) Representative results of fluorescent in situ hybridization and the enlarged chromosome 10 in each patient. Yellow arrows denote the deletions. Of the enlarged chromosomes, the left one is intact and the right one is affected, and white bands indicate the position of the centromere. (e) Results obtained with the Agilent Human Genome CGH Microarray $244 \mathrm{~K}$ in each patient. Black arrows indicate each CNV. (f) Scheme of the region around the deletions, genes and miRNA. Open vertical bars indicate the deletions of the current patients and grey vertical bars indicate the deletions in the previous reports. Thin horizontal arrows indicate genes and their directions. Vertical dashed lines indicate the SRO the current patients, and the vertical shading indicates the SRO of the cases reported previously. ${ }^{8}$ A full color version of this figure is available at the Journal of Human Genetics journal online. 
Table 1 Summary of clinical features of current and previously reported cases with a deletion at 10p12.1-p11.23

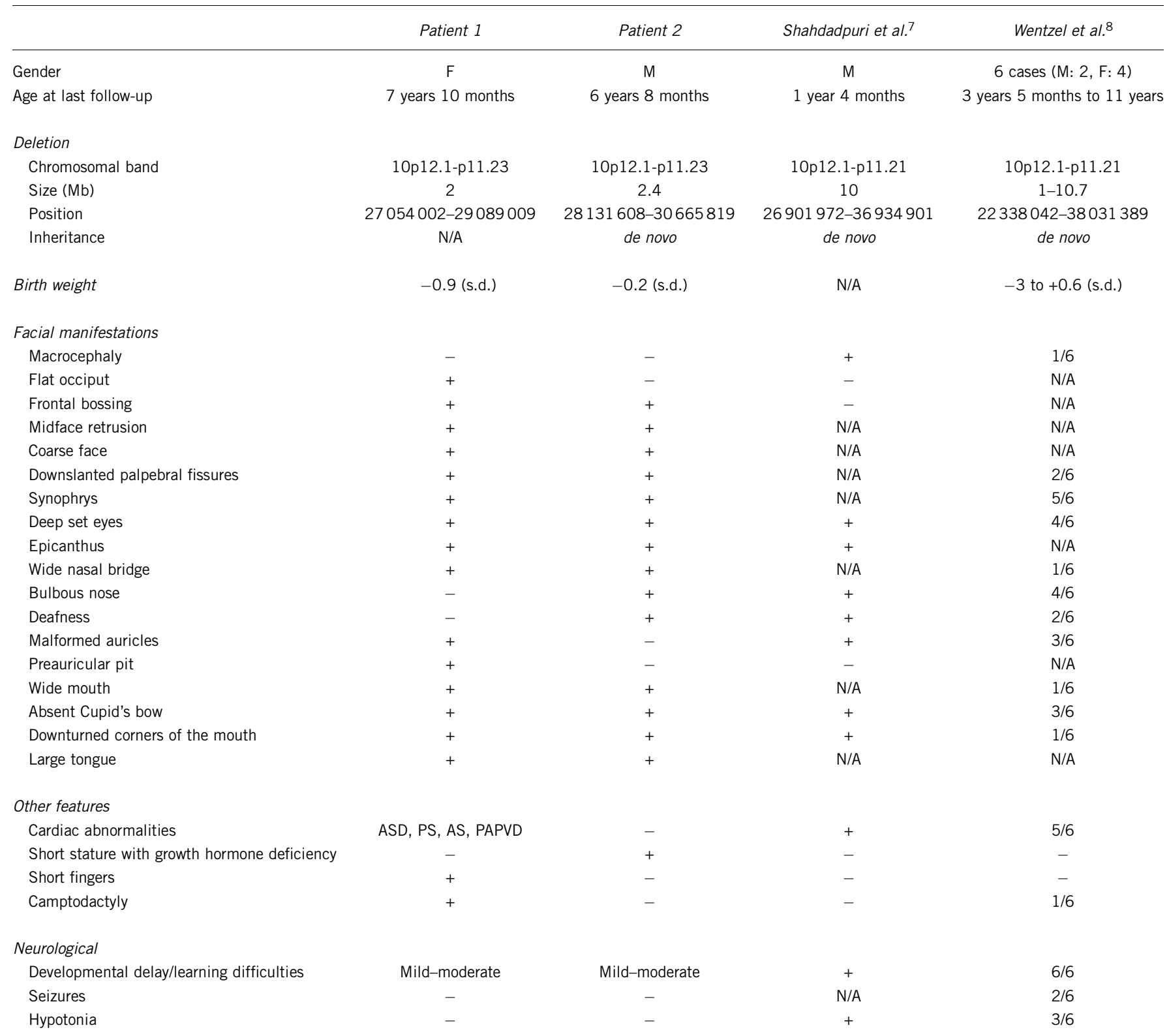

Abbreviations: +, present; -, absent; AS, aortic stenosis; ASD, atrial septal defect; F, female; M, male; N/A, not applicable; PAPVD, partial anomalous pulmonary venous connection; PS, pulmonary stenosis.

\section{Fluorescent in situ hybridization}

Fluorescent in situ hybridization was performed as described previously, ${ }^{6}$ using BACs located around the region of interest as probes.

\section{Cephalometric analysis}

A cephalometric analysis was performed using lateral cephalometric radiographs to determine the extent of the skeletal and/or dental malocclusion. Traditional landmarks, linear and angular measurements, were used in this study. ${ }^{10-12}$ Every measurement of the cephalogram was performed by one experienced orthodontist. To minimize error, each measurement was repeated at least twice. Every error in the measurement was within $\pm 0.5^{\circ}$ or $0.5 \mathrm{~mm}$. These measurements were compared with sex, race and age-matched controls. The norms of the Japanese head circumference by Ishikawa et al., ${ }^{13}$ the Japanese norms of $\mathrm{N}-\mathrm{Me}$ (nasion menton), $\mathrm{N}-\mathrm{ANS}$ ( $\mathrm{N}$-anterior nasal spine), ANS-Me, N-S (N-sella trucica), S-Ba (S-basion), ANS-PNS (ANS-posterior anasal spine ), Ar-Go (articulare-gonion) and Go-Pog (Go-pogonion) by Masaki, ${ }^{14}$ the Japanese norms of SNA angle (angle between SN and NA), U1 (tip of the maxillary central incisor crown) to FH (frankfort horizontal plane), facial angle, SNB angle (angle between SN and NB), mandibular plane angle, gonial angle, L1 (tip of the mandibular central incisor crown) to mandibular plane angle and A-B plane angle by lizuka, ${ }^{11}$ and the norm of N-S-Ar reported by Björk ${ }^{15}$ were employed (Table 2). Linear measurements of lateral cephalometric variables, based on Coben's coordinate system ${ }^{10}$ using the Frankfort horizontal plane, were compared with the Japanese norms (Table 2).

\section{RESULTS}

\section{Cytogenetic analyses}

The aCGH analysis using the in-house Whole Genome Array-4500 detected partially overlapping heterozygous deletions at $10 \mathrm{p} 11$. 
Table 2 Results of the cephalometric measurements

\begin{tabular}{|c|c|c|c|c|c|c|c|c|c|c|c|c|}
\hline \multirow[b]{2}{*}{ Measurement items (angular) } & \multicolumn{2}{|c|}{$\begin{array}{c}\text { IIC } 6 \text { years } \\
3 \text { months } \pm 1.8\end{array}$} & \multirow[b]{2}{*}{ Patient 1} & \multirow[b]{2}{*}{ Patient 2} & \multirow{2}{*}{\multicolumn{2}{|c|}{ Measurement items (linear) }} & \multicolumn{2}{|c|}{$\begin{array}{l}7 \text { years } \\
\text { (male) }\end{array}$} & \multicolumn{2}{|c|}{$\begin{array}{l}7 \text { years } \\
\text { (female) }\end{array}$} & \multirow[b]{2}{*}{ Patient 1} & \multirow[b]{2}{*}{ Patient 2} \\
\hline & Mean & s.d. & & & & & Mean & s.d. & Mean & s.d. & & \\
\hline SNA & 81.35 & 2.78 & 82.7 & 77.11 & Depth & $\mathrm{Ba}-\mathrm{N}(\mathrm{mm})$ & 86.5 & 3.1 & 86.4 & 3.4 & 84.49 & 88.7 \\
\hline SNB & 76.38 & 2.08 & 77.97 & 79.8 & & N-S (\%) & 73.6 & 2 & 71.7 & 2.5 & 70.49 & 69.9 \\
\hline MP angle & 31.13 & 5.19 & 26.46 & 29.52 & & S-Ptm (\%) & 21.5 & 2.7 & 22.1 & 3.1 & 18.18 & 18.54 \\
\hline Gonial angle & 130.41 & 4.31 & 110.71 & 119.77 & & $\mathrm{Ba}-\operatorname{Ar}(\%)$ & 12.6 & 2.1 & 13.8 & 2.1 & 13.61 & 20.69 \\
\hline Ramus inclination & 80.81 & 4.41 & 95.75 & 89.76 & & Ar-Go (\%) & 6.1 & 3.6 & 5.2 & 2.4 & 4.51 & 0.18 \\
\hline Occlusal plane angle & 14.25 & 4.28 & 6.89 & 15.18 & Height & $\mathrm{N}-\mathrm{M}(\mathrm{mm})$ & 106.4 & 3.9 & 105.7 & 3.4 & 105.26 & 109.41 \\
\hline $\mathrm{U} 1$ to $\mathrm{FH}$ & 96.35 & 4.65 & 126.89 & 108.1 & & N-ANS (\%) & 44.6 & 1.2 & 44.9 & 1.7 & 43.14 & 40.19 \\
\hline \multirow[t]{2}{*}{ L1 to MP } & 84.42 & 6.18 & 106.62 & 87.77 & & ANS-Me (\%) & 55.6 & 1.3 & 55 & 1.8 & 55.76 & 59.07 \\
\hline & & & & & & U1-L1 (\%) & 2.2 & 0.9 & 1.3 & 0.8 & -13.95 & 29.22 \\
\hline
\end{tabular}

Abbreviations: ANS, anterior nasal spine; Ar, articulare; Ba, basion; FH, frankfort horizontal plane; Go, gonion; L1, tip of mandibular incisor crown; Me, menton; MP, mandibular plane; N, nasion; Po, Pogonion; Ptm, Pterygomaxillary fissure; S, sella trucica; SN, SN plane; SNA, angle between SN and NA; SNB, angle between SN and NB; U1, tip of maxillary central incisor crown.

a

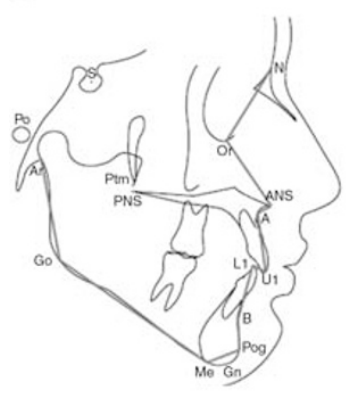

b

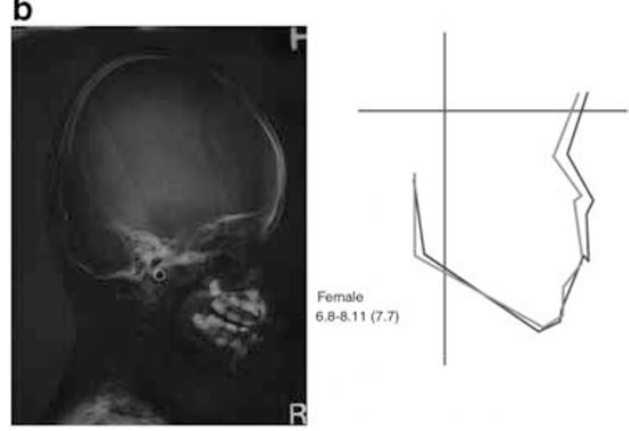

C

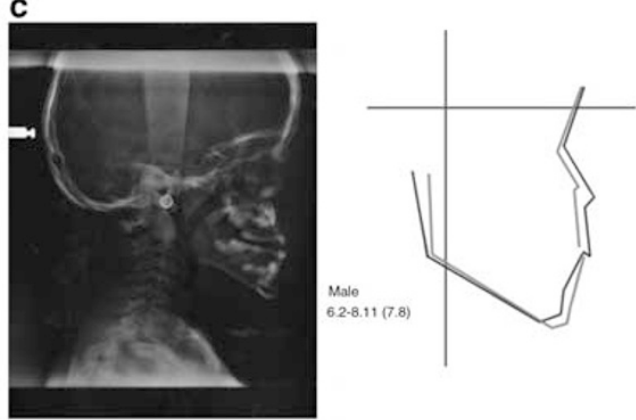

Figure 2 (a) The positions of all cephalometric measurements. (b, c) Lateral cephalogram and profilegrams of patient 1 and 2 , respectively. The red line in the profilegrams depicts the patient. The blue line is the Japanese age- and sex-matched norm. A, point A; ANS, anterior nasal spine; Ar, articulare; B, point B; Ba, basion; Gn, gnathion; Go, gonion; L1, tip of mandibular central incisor crown; Me, menton; N, nasion; Pog, pogonion; PNS, posterior nasal spine; S, sella trucica; U1, tip of maxillary central incisor crown. A full color version of this figure is available at the Journal of Human Genetics journal online.

23-p12.1 in both of the patients (Figure 1c), and fluorescent in situ hybridization confirmed the deletions (Figure 1d). In patient 2, fluorescent in situ hybridization detected the presence of two copies in both the parents, suggesting that the CNV was de novo (data not shown). The oligonucleotide array determined the precise sizes of the deletions (Figure 1e), which are described as follows: arr 10 12.1p11.23(27054002-29089009) $\times 1$ in patient 1 and arr 10p12.1p11.23(28 131608-30665819) $\times 1$ in patient 2 . The deletions included 12 and 16 RefSeq genes in patient 1 and 2, respectively. The smallest region of overlapping (SRO) of the deletions is $957 \mathrm{~kb}$ at 10p11.23-p12.1 and contains four RefSeq genes, ARMC4, MPP7, WAC and $B A M B I$, as well as two microRNAs (Figure 1f). We also estimated all the CNVs detected by the BAC and oligonucleotide arrays other than the deletions at 10p11.23-p12.1 as non-pathogenic, in reference to the databases of the genomic variants; for example, Database of Genome Variation or Segmental Duplication Database (data not shown).

\section{Intraoral and skeletal characteristics}

No primary teeth of the patients had morphological defects, and no failure of eruption of the permanent teeth was found. As of the large tongue, the patients exhibited an anterior open bite due to the proclined anterior teeth (Figures $1 \mathrm{a}$ and $\mathrm{b}$ ). Using traditional landmarks, linear and angular measurements, cephalometric measurements of the patients were performed (Figure 2a, Table 2). ${ }^{10-12}$ Cephalometric radiographs showed no abnormalities in anatomy. Certainly, the significant craniofacial dysmorphology of our patients was characterized by reductions in the cranial base length $(\mathrm{S}-\mathrm{N})$ and midfacial depth (Ptm-A (Pterygomaxillary fissure-A)) as compared with the Japanese norms. ${ }^{16,17}$ Profilegrams also showed apparent maxillary retrognathism (Figures $2 \mathrm{~b}$ and $\mathrm{c}$ ). The angle of the mandible (gonial angle) was less than 2 s.d. smaller. On the other hand, the ramus inclination was more than 2 s.d. higher in our patients. The proclination of both maxillary and mandibular incisors (U1 to FH and L1 to mandibular plane) was seen.

\section{DISCUSSION}

Recent advances in aCGH now allow for the detection of smaller CNVs with precise localization of the breakpoints and better delineation of the corresponding phenotype. Although this method is now important in identifying genes having a major role in the pathogenesis of congenital anomalies and mental retardation, it has not been used much in the field of dentistry. Through the screening of 536 patients with undiagnosed MCA/MR using aCGH, we detected overlapping 
interstitial deletions at 10p11.23-p12.1 in two patients. ${ }^{6}$ The SRO was narrowed down to a $957-\mathrm{kb}$ area after the fine mapping of deleted regions, and the two cases revealed many common clinical features, prompting us to define a genotype-phenotype correlation. Many of the prominent clinical characteristics in both cases were observed in the maxillofacial region: frontal bossing, midface retrusion, coarse face, downslanted palpebral fissures, synophrys, deep set eyes, epicanthus, wide nasal bridge, wide mouth, absent Cupid's bow, everted vermilion of the upper lip, downturned corners of the mouth, large tongue and short cranial base length. Additionally, the large tongue causes several symptoms of malocclusion; for example, labioclination of maxillary incisors, open bite and mild mandibular prognathism as previously reported. ${ }^{18,19}$ However, no reports have elaborated on the deformity of the facial anomalies.

To our knowledge, several cases of overlapping heterozygous deletions involved in 10p11-p12 have been reported. ${ }^{7,8}$ All of them revealed developmental delay and also seem to have similar characteristic phenotypes to our patients, although their maxillofacial features are not stated clearly (Table 1). For example, deep set eyes were observed in both of our cases and five of the seven previously reported cases, suggesting this to be a common feature associated with the 10p11-p12 deletion and the phenotype resulting from midface retrusion. Although not described clearly, several features; for example, coarse face, large tongue, downturned corners of the mouth, wide mouth or absent Cupid's bow, were also probably observed in the previous cases. Thus, to assess the maxillofacial characteristics, we performed a quantitative examination of craniofacial characteristics through an orthodontics analysis; for example, cephalometric analysis in subjects with the 10p11-12 interstitial deletion. Subsequent maxillofacial characterization using cephalometric measurements demonstrated an obvious reduction in cranial base length and midfacial depth. The current study is the first to quantitatively measure the craniofacial characteristics in a subject with a $10 \mathrm{p}$ interstitial deletion.

The SRO of our cases contains four protein-coding genes; ARMC4, $M P P 7, W A C$ and $B A M B I$, and the latter two genes are also contained in the SRO of the reported cases. ${ }^{8}$ WAC is reported to be one of the candidates for the developmental delay based on the evidences of Drosophila model, ${ }^{8}$ although its function remains unknown. BAMBI encodes a transmembrane glycoprotein and is suggested to have a role in modulating the signaling range of the transforming growth factor- $\beta$ family during early embryogenesis; ${ }^{20}$ however, the Bambid ${ }^{\text {del/del }}$ mutant mice did not exhibit any obvious defect in the skeletal and limb development and postnatal survival. ${ }^{21}$ ARMC4 was identified as regulators of ciliogenesis, but the other functions remain unknown. ${ }^{22}$ MPP7 belonging to the membrane-associated guanylate kinases are reported to form a complex, promoting epithelial cell polarity and tight-junction formation. ${ }^{23}$

The concordance of both the phenotypes and the genotypes characterize the clinical condition corresponding to the deletion at the SRO. Thus, the mutation of a single gene involved in the SRO may also cause phenotypes identical to the patients with the deletion at 10p11.23-p12.1. However, other genetic modifiers also potentially affect phenotypes. For example, cardiac abnormalities are frequently observed in patients whose deletions at 10p11.23-p12.1 do not overlap (Table 1). Other genetic components, for example, cryptic CNVs, at positions other than 10p11.23-p12.1, may modify the phenotypes in an additional or epistatic manner. ${ }^{24}$

A number of rapidly developed cytogenetic and molecular approaches have been applied to the screening or investigation of various congenital disorders. However, a precise phenotypic assessment as well as high-performance genetic analysis is required to establish a correlation between genotypes and phenotypes. The current study is the first trial to examine quantitatively the craniofacial characteristics of patients with a heterozygous deletion at 10p11.23-p12.1. As the major features were observed in the maxillofacial region, the craniofacial assessment probably presents good evidence for diagnosing not only patients with the 10p11.23-p12.1 deletion, but also a patient with a mutation of the corresponding gene(s) in the SRO, WAC and/or $B A M B I$. Precise clinical assessment will be helpful to diagnose potential patients with the same genetic cause and to establish a correlation between genotypes and phenotypes.

\section{CONFLICT OF INTEREST}

The authors declare no conflict of interest.

\section{ACKNOWLEDGEMENTS}

This study was supported in part by Grants-in-Aid for Scientific Research and Scientific Research on Priority Areas, and a Global Center of Excellence (GCOE) Program for International Research Center for Molecular Science in Tooth and Bone Diseases from the Ministry of Education, Culture, Sports, Science and Technology, Japan; a Health and Labour Sciences Research Grant by the Ministry of Health, Labour and Welfare, Japan; and a grant from the New Energy and Industrial Technology Development Organization (NEDO). We are grateful to Ayako Takahashi and Rumi Mori for technical assistance.

\section{Web Resources}

UCSC Genome Bowser, March 2006 build (NCBI36/hg18), http://genome.ucsc.edu/ Database of Genome Variation (Nov. 02, 2010), http://projects.tcag.ca/variation/ Segmental Duplication Database, http://projects.tcag.ca/humandup/ Segmental Duplication Database, http://humanparalogy.gs.washington.edu/.

1 Centers for Disease Control and Prevention (CDC). Surveillance Summaries temporal Trends in the Incidence of Birth Defects - United States. Morb. Mortal. Wkly Rep. 46, 1171-1176 (1997).

2 Hunter, A.G. Outcome of the routine assessment of patients with mental retardation in a genetics clinic. Am. J. Med. Genet. 90, 60-68 (2000).

3 Lary, J. M. \& Paulozzi, L. J. Sex differences in the prevalence of human birth defects: a population-based study. Teratology 64, 237-251 (2001).

4 Lisi, A ., Botto, L.D., Rittler, M ., Castilla, E ., Bianca, S ., Bianchi, F . et al. Sex and congenital malformations: an international perspective. Am. J. Med. Genet. A 134, 49-57 (2005).

5 Miller, D.T., Adam, M.P., Aradhya, S., Biesecker, L.G., Brothman, A.R., Carter, N.P. et al. Consensus statement: chromosomal microarray is a first-tier clinical diagnostic test for individuals with developmental disabilities or congenital anomalies. Am. J. Hum. Genet. 86, 749-764 (2010).

6 Hayashi, S., Imoto, I., Aizu, Y., Okamoto, N., Mizuno, S., Kurosawa, K. et al. Clinical application of array-based comparative genomic hybridization by two-stage screening for 536 patients with mental retardation and multiple congenital anomalies. J. Hum. Genet. 56, 110-124 (2011).

7 Shahdadpuri, R., de Vries, B., Pfundt, R., de Leeuw, N. \& Reardon, W. Pseudoarthrosis of the clavicle and copper beaten skull associated with chromosome 10p11.21p12.1 microdeletion. Am. J. Med. Genet. A 146, 233-237 (2008).

8 Wentzel, C., Rajcan-Separovic, E., Ruivenkamp, C. A., Chantot-Bastaraud, S., Metay, C., Andrieux, J. et al. Genomic and clinical characteristics of six patients with partially overlapping interstitial deletions at 10p12p11. Eur. J. Hum. Genet. 19, 959-964 (2011).

9 Inazawa, J., Inoue, J. \& Imoto, I. Comparative genomic hybridization (CGH)-arrays pave the way for identification of novel cancer-related genes. Cancer. Sci. 95, 559-563 (2004).

10 Coben, S. E. The integration of facial skeletal variants. Am. J. Orthod. 41, 407-434 (1955).

11 lizuka, T. Roentgencephalometric analysis of craniofacial growth in Japanese children. J. Stomatol. Soc. Jpn 25, 18-30 (1958).

12 Sakamoto, T. A study on the development changes of dentofacial complex of the Japanese with special reference to sella turcica. J. Jpn Orthod. Soc. 18, 1-17 (1959).

13 Ishikawa, T., Furuyama, M., Ishikawa, M., Ogawa, J. \& Wada, Y. Growth in head circumference from birth to fifteen years of age in Japan. Acta. Pediatr. Scand. 76, 824-828 (1987).

14 Masaki, F. [The longitudinal study of morphological differences in the cranial base and facial structure between Japanese and American whites]. Nippon Kyosei Shika Gakkai Zasshi. 39, 436-456 (1980).

15 Björk, A. The face in profile: an anthropological x-ray investigation on Swedish children and conscripts. Am. J. Orthod. 34, 691-699 (1948). 
16 Mitani, H. A follow-up study growth increment and rate in the human face during puberty, part I: study of growth increment [in Japanese]. Jpn J. Orthod. 31, 307-318 (1972).

17 Lin, T. Z. [Postero-anterior cephalometric features in Japanese children with malocclusion]. Kokubyo Gakkai Zasshi. 56, 361-80 (1989).

18 Ngan, P. \& Fields, H. W. Open bite: a review of etiology and management. Pediatr. Dent. 19, 91-98 (1997).

19 Liu, Z.J., Shcherbatyy, V., Gu, G . \& Perkins, J.A. Effects of tongue volume reduction on craniofacial growth: a longitudinal study on orofacial skeletons and dental arches. Arch. Oral. Biol. 53, 991-1001 (2008).

20 Onichtchouk, D., Chen, Y. G., Dosch, R., Gawantka, V., Delius, H., Massagué, J. et al. Silencing of TGF-beta signalling by the pseudoreceptor BAMBI. Nature 401, 480-485 (1999).
21 Chen, J., Bush, J. O., Ovitt, C. E., Lan, Y. \& Jiang, R. The TGF-beta pseudoreceptor gene Bambi is dispensable for mouse embryonic development and postnatal survival. Genesis 45, 482-486 (2007).

22 Lonergan, K. M., Chari, R., Deleeuw, R. J., Shadeo, A., Chi, B., Tsao, M. S. et al. Identification of novel lung genes in bronchial epithelium by serial analysis of gene expression. Am. J. Respir. Cell. Mol. Biol. 35, 651-661 (2006).

23 Stucke, V. M., Timmerman, E., Vandekerckhove, J., Gevaert, K. \& Hall, A. The MAGUK protein MPP7 binds to the polarity protein hDIg1 and facilitates epithelial tight junction formation. Mol. Biol. Cell. 18, 1744-1755 (2007).

24 Girirajan, S., Rosenfeld, J.A., Cooper, G.M., Antonacci, F., Siswara, P., Itsara, A . et al. A recurrent $16 \mathrm{p} 12.1$ microdeletion supports model for severe developmental delay. Nat. Genet. 42, 203-209 (2010). 